Ann. Zootech., I977, 26 (4), 595-6rз.

\title{
L'excrétion biliaire chez le Porc. Influence des repas et rôle éventuel de récepteurs oddiens dans le contrôle du débit cholédocien
}

\author{
J. P. LAPLACE et M. A. OUAISSI \\ avec la collaboration technique de C. GERMAIN et A. ROGER \\ Labovatoive de Physiologie de la Nutrition \\ Centre national de Recherches zootechniques, I.N.R.A., \\ $7835^{\circ}$ Jouy en Josas (France)
}

\begin{abstract}
Résumé
Le débit de la sécrétion biliaire, collectée par fistulation de l'about hépatique du cholédoque sectionné, a été mesuré chez I4 porcs de race Large White pesant de 57 à $63 \mathrm{~kg}$, la bile étant réintroduite à débit constant tout au long du nycthémère soit dans l'about intestinal du cholédoque, irriguant ainsi le trajet sphinctérien, soit directement dans la lumière duodénale. La variabilité du débit biliaire d'un jour à l'autre pour un mêtme animal, et d'un individu à l'autre apparaît comme relativement importante sans qu'une relation régulière puisse être établie avec le niveau d'ingestion. Les repas semblent responsables d'une certaine rythmicité du débit biliaire horaire au long des $24 \mathrm{~h}$. D'une manière générale, ce débit se trouve téduit d'une façon plus ou moins précoce et prolongée au moment et/ou à la suite des repas, particularité qui pourrait être reliée à la constance du débit de restitution de la bile à l'animal. Les variations brèves du débit dans la période périprandiale immédiate suggèrent que l'évacuation vésiculaire ne survient que lorsque le trajet Oddien est irrigué par la bile restituée à l'animal. Néanmoins cette évacuation ne paraît porter que sur un volume relativement limité. Par ailleurs, les quantités totales de bile produites (de l'ordre de 21 en $24 \mathrm{~h}$ ) sont significativement plus élevées lors de réintroduction stss-oddienne. Ces particularités viennent étayer l'hypothèse d'un rôle fonctionnel des formations réceptrices localisées au niveau du trajet sphinctérien (Oddi) à l'égard du fonctionnement de la vésicule biliaire d'une part et du contrôle de la cholérèse d'autre part.
\end{abstract}

\section{Introduction}

Dans un précédent travail relatif à la dynamique de l'excrétion biliaire chez le Porc (LAPLACE, 1976b), diverses observations nous ont conduit à envisager l'existence de relations réflexes entre le sphincter d'Oddi et la vésicule biliaire. Une telle activité a déjà été signalée par WYATT, BELZER et DUNPHy (I967) et 
par Hopton (I973). L'ablation de la vésicule biliaire chez le Chien conduit entre autres séquelles à un état béant du sphincter d'Oddi (Shriner, I967).

Chez le Porc soumis à dérivation biliaire en amont du sphincter, la vésicule est apparue à l'examen nécropsique, comme probablement infonctionnelle (LAPLA. $\mathrm{CE}, \mathrm{I} 976 \mathrm{~b}$ ). On peut ainsi s'interroger sur l'origine de cette influence de la fistulation du canal cholédoque. Une perception de la vacuité du trajet sphinctérien et du cholédoque terminal, dont DEKHKanov (I974) a montré l'extrême richesse en formations réceptives, pourrait être en cause. Plus précisément la non stimulation de ces récepteurs entraînerait l'involution vésiculaire.

Face à ces problèmes soulevés par des observations expérimentales et nécropsiques antérieures, il nous a paru utile de décrire le débit biliaire cholédocien en $24 \mathrm{~h}$ en fonction du nombre de repas et de la quantité ingérée, respectivement dans des conditions d'exclusion totale du sphincter d'Oddi et lors d'irrigation du trajet sphinctérien par la bile restituée à l'animal. Une éventuelle confirmation de la suppression de la fonction vésiculaire lors d'exclusion fonctionnelle du sphincter d'Oddi autoriserait à assimiler le débit cholédocien mesuré dans ces conditions à un débit de bile hépatique.

\section{Matériel et méthodes}

Au total I 4 pores de race Large White, mâles castrés, ont été préparés chirurgicalement pour cette étude. Ils ont été utilisés entre un poids vif moyen à l'opération de $56,9 \pm I, I \mathrm{~kg}$ et un poids vif moyen d'abattage de $62,7 \pm 2,0 \mathrm{~kg}$. Ils ont été hébergés pendant la totalité de la période expérimentale dans des cages analogues à des cages à métabolisme et ont reçu un aliment standard de croissance sous forme de farine diluée dans 2 fois son poids d'eau. La distribution de l'aliment a été effectuée soit 2 fois par jour, à heures fixes ( 9 -I 7 h) ou variables, soit I seule fois par jour à $I 7 \mathrm{~h}$.

\section{I. - Préparation chirurgicale}

Le canal cholédoque est mis à jour à la faveur d'une laparotomie sous anesthésie générale, induite au penthiobarbital I.V. (Nesdonal, R) et entretenue au méthoxyflurane (Penthrane, R) appliqué au masque.

Pour I I porcs, a été utilisé le principe d'une dérivation biliaire cholédocienne, avec réintroduction directement dans la lumière duodénale. Pour cela, un tube de silicone médical (Silastic, R) d'un calibre int. /ext. de 3,I8-6,35 $\mathrm{mm}$ a été introduit dans 1'about hépatique du canal cholédoque sectionné. L'utilisation de tubes d'un calibre intérieur plus faible, testée lors d'expériences préliminaires, conduit en effet à plus ou moins brève échéance à une rétention biliaire chez des porcs de ce poids. L'about intestinal du cholédoque est ligaturé quelques $\mathrm{mm}$ en amont du sphincter d'Oddi. Un tube identique est mis en place dans le duodénum, à environ $\mathrm{r} 2 \mathrm{~cm}$ du pylore, pour permettre une réintroduction continue de bile. Trois de ces I porcs ont été réservés aux enregistrements avec des distributions d'aliment à horaites variables.

Pour 3 autres pores, la dérivation biliaire au niveau de l'about hépatique du cholédoque est réalisée de façon semblable. Par contre, la réintroduction de la bile est effectuée grâce à l'implantation dans l'about intestinal du cholédoque d'un 
tube identique dont l'extrémité est localisée à 5 à $10 \mathrm{~mm}$ en amont de la formation oddienne. De la sorte, 1e trajet sphinctérien peut être constamment irrigué par la bile rendue à l'animal.

Les tubes de silicone mis en place dans les abouts intestinal et hépatique du canal cholédoque, ou dans le duodénum, ont été préalablement préparés par la réalisation d'une fine collerette d'élastomère de silicone, tout comme pour une intubation vasculaire (LAPLACE, I972). Ce mince bourrelet (situé à $5 \mathrm{~mm}$ de l'extrémité du tube cholédocien ou $2 \mathrm{~cm}$ de celle du tube duodénal) limite un recul éventuel grâce à la ligature placée immédiatement en arrière : ligature circonférentielle modérément serrée sur le cholédoque; suture en bourse sur la paroi duodénale. Ces tubes font par ailleurs l'objet d'un ancrage solide sur le méso-duodénum, évitant toute mobilisation traumatisante. Ils sont extériorisés à mi-hauteur du flanc de l'animal et à nouveau fixés à un bandage ceinturant 1'abdomen du porc.

\section{II. - Mesures du débit de bile}

Le tube par lequel s'écoule la sécrétion biliaire est prolongé par une tubulure de calibre identique jusqu'à un point fixe extérieur à la cage de l'animal. Cette tubulure est guidée par des liaisons élastiques à la paroi de la cage, de façon à accompagner les mouvements des porcs. L'ouverture terminale au niveau de laquelle est observé le goutte à goutte de la bile est située sur le même plan horizontal que le plancher sur lequel repose l'animal en décubitus. La bile s'écoule ainsi librement sans aspiration par siphonnage, ni surpression au niveau des voies biliaires.

La bile est ainsi recueillie 24 h sur 24 sur un collecteur de fractions dont la rotation intervient toutes les $5 \mathrm{mn}$. Une cellule photo-électrique permet éventuellement, pour des périodes brèves (3o mn consécutives au repas), d'enregistrer simultanément le débit biliaire en comptage de gouttes par unité de temps plus faible ( $\mathrm{mn}$ ). Les volumes collectés par $5 \mathrm{mn}$ sont, après lecture, destinés à la réintroduction. Celle-ci est assurée par une pompe à régulation électronique (type Transomega II A), avec un débit constant. Ce débit $/ \mathrm{mn}$ est, pour chaque nycthémère expérimental, calqué sur le débit moyen $/ \mathrm{mn}$ au cours du nycthémère précédent. La bile est systématiquement stockée sous huile de paraffine afin d'éviter tout contact prolongé avec 1'air.

\section{Résultats}

\section{I. - Débit biliaire cholédocien lors d'exclusion oddienne}

a) Aspects quantitatifs.

Chez les porcs préparés avec fistule cholédocienne et réintroduction intestinale directe, les quantités totales de bile émise en $24 \mathrm{~h}$ sont de l'ordre de $2000 \mathrm{ml}$ (tableau I). Ce volume total moyen produit par $24 \mathrm{~h}$ ne diffère pas significativement entre les deux groupes de données correspondant l'un aux enregistrements effectués lors de double distribution alimentaire par jour, 1'autre aux données obtenues 
TABIEAU I

Quantités totales moyennes (ml) de bile collectée en 24 h chez des porcs à fistulation cholédocienne et réintroduction intestinale (sphincter d'Oddi exclu). Les distributions d'aliment interviennent systématiquement $\dot{a}_{9} h$ et $I_{7} h$ ou $\dot{a} I_{7} h$ lors de repas unique.

Mean total volume (ml) of bile collected during a 24 h period in pigs fitted with a fistula of the choledochus and having bile returned directly into the duodenum. Meal time $=9$ a.m. and 5 p.m. or 5 p.m. only.

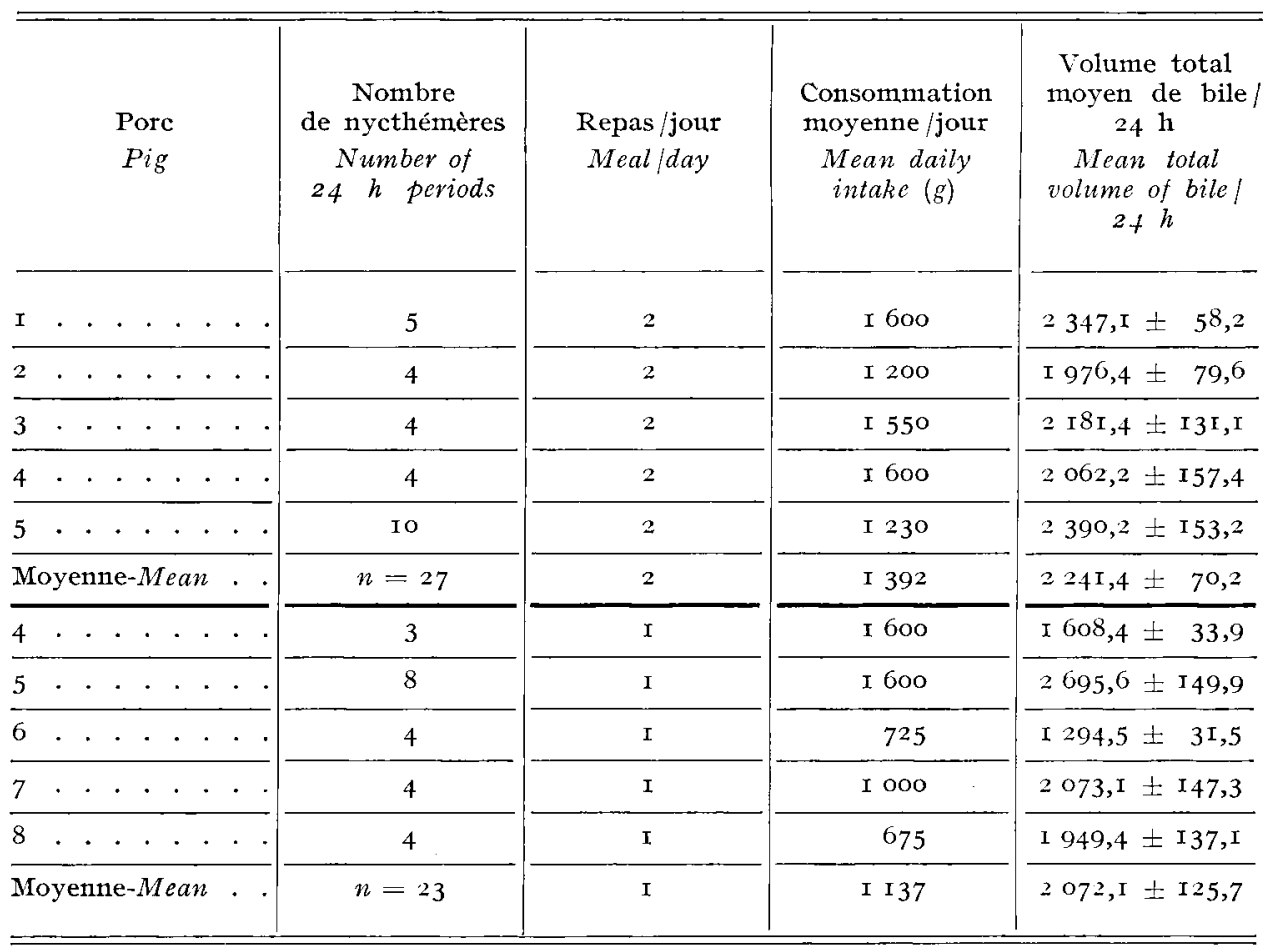

lors de distribution unique. 'Toutefois lorsqu'on procède à cette même comparaison chez un même animal (cas des pores 4 ou 5 ) on peut observer une différence non négligeable (porc $4, t=2,40 p<0$, xo) entre le volume moyen de bile par $24 \mathrm{~h}$ en situation de double distribution et celui qui est observé en période de repas quotidien unique.

La variabilité des quantités quotidiennes de bile, évaluée dans ces deux groupes (27 nycthémères de 5 porcs $/ 23$ nycthémères de 5 porcs), atteint $16,3 \mathrm{p}$. cent (2 repas/jour) et 29 , I p. cent ( $\mathrm{I}$ repas/jour). Il n'y a pas cependant de différence significative entre les volumes moyens par $24 \mathrm{~h}$ produits par chacun des porcs comparés deux à deux, à l'exception du porc 6 dont la production de bile est faible $(t=5,83 \longrightarrow p<0,0 \mathrm{r})$. Cette dernière particularité ne peut être rattachée à la faible ingestion d'aliment de cet animal comme l'indiquent les résultats du porc 8.

L'influence de la variation du niveau d'ingestion, testée chez le porc 5 avec double distribution alimentaire quotidienne pour des niveaux variant de 800 à I $600 \mathrm{~g} /$ jour s'avère significative selon la régression linéaire du débit en $24 \mathrm{~h}$ 
en fonction de l'ingéré/jour $(y=\mathrm{I}, 50 x+544,4 ; r=0,90)$. Mais cette même relation n'est pas retrouvée chez le Porc 8 avec distribution unique de 300 à $\mathrm{I}$ ooo $\mathrm{g} /$ jour $(y=0,2 \mathrm{I} \quad x+\mathrm{I} 662,6 ; \gamma=0, \mathrm{I} 4)$.

\section{b) Variabilité du débit cholédocien au cours des 24 heures}

Les diverses particularités individuelles ci-dessus signalées ne sont pas plus étonnantes que ne peut surprendre l'irrégularité du débit cholédocien tel qu'il a été mesuré, soit par fraction de $5 \mathrm{mn}$. La figure I illustre cet aspect dans le cas du porc 4 , et les coefficients de variation du débit au long des $24 \mathrm{~h}$ en témoignent:

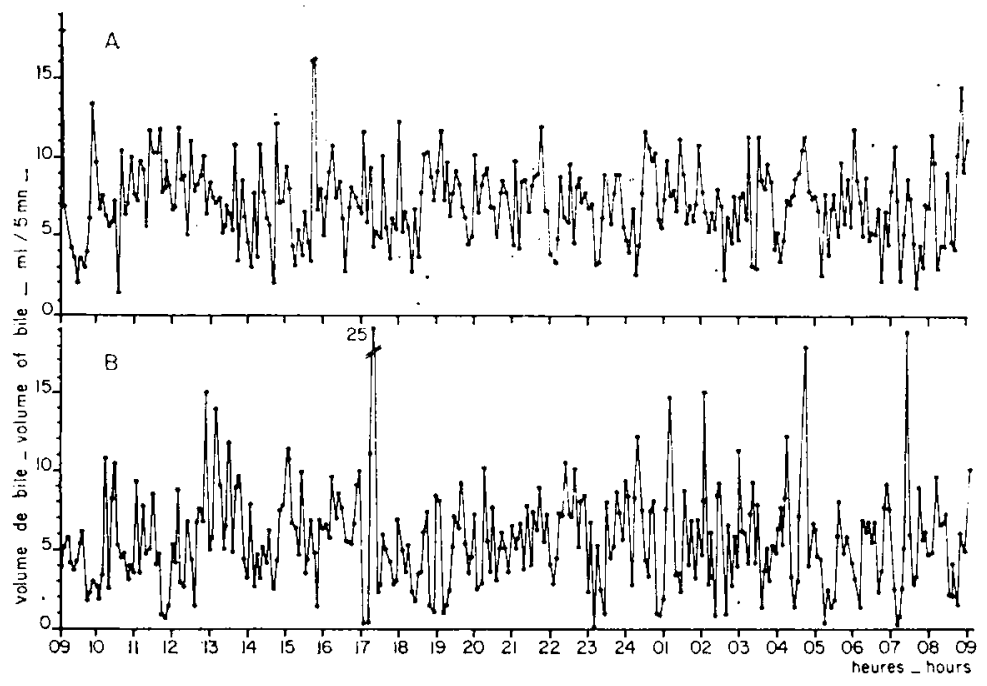

FIG. I. -- Évolution au cours du nycthémère du débit biliaire cholédocien moyen par 5 mn chez le porc \& à sphincter d'Oddi exclu (réintroduction intestinale divecte).

En $A:$ Moyenne de 4 enregistrements avec 2 repas ( $800 \mathrm{~g}$ d'aliment sec) à 9 et $\mathrm{I} 7 \mathrm{~h}$. En $B:$ Moyenne de 3 enregistrements avec I repas ( $1600 \mathrm{~g})$ à $I_{7} \mathrm{~h}$.

Mean bile flow per $5 \mathrm{mn}$ from a choledochus fistulated pig $\left(n^{\circ} \quad 4\right)$, bile being veturned directly into the duodenum, throughout the 24 heriod.

A: Mean curve based on 4 days with 2 meals $(800 \mathrm{~g}$ each) per day at 9 a.m. and 5 p.m.

B: Mean curve based on 3 days with $x$ meal ( $1600 \mathrm{~g}$ ) at 5 p.m.

on en registre ainsi, respectivement pour une courbe moyenne de 4 enregistrements de $24 \mathrm{~h}$ avec 2 repas de $800 \mathrm{~g}$ par jour et pour une courbe moyenne de 3 enregistrements avec I seul repas de I $600 \mathrm{~g}$ par jour, les indications de débit ci-après :

2 repas/jour : 7,I5 $\pm 0, \mathrm{I}_{5} \mathrm{ml} / 5 \mathrm{mn}$ - coefficient de variation $36,0 \mathrm{p}$. cent, I repas/jour : $5,74 \pm 0, \mathrm{I} 9 \mathrm{ml} / 5 \mathrm{mn}$ - coefficient de variation $57,3 \mathrm{p}$. cent. 
Cela étant, il est plus aisé d'examiner l'évolution du débit biliaire cholédocien moyen tout au long du nycthémère sur la base des volumes émis par heure, bien que la variabilité teste importante. Le coefficient de variation du débit au cours d'une même heure du cycle de $24 \mathrm{~h}$, chez 5 porcs soumis à réintroduction intestinale, varie selon la tranche horaire considérée :

I repas/jour : de $I 6,8$ à $40 \mathrm{p}$. cent sur la base de 27 nycthémères, 2 repas/jour : de 25,3 à 40,8 p. cent sur la base de 24 nycthémères.

Par ailleurs, la variabilité globale du volume de bile émis par heure, calculée porc par porc, est indiquée avec la valeur moyenne de ce débit dans le tableau 2 .

\section{TABLEAU 2}

Volume de bile émis par heure chez le Porc selon le site de réintroduction de la bile collectée et selon. le nombre de repas distribués par jour: moyenne (en $\mathrm{ml}$ ) (x) écart-type de la moyenne (s $\overline{\mathbf{x}})$ coefficient de variation en p. cent (C.V.) nombre d'heures de mesure concernées (n).

Volume of bile produced per hour by the Pig according to the way of bile vestitution and to the number of meals allotted per day: mean $(\overline{\mathrm{x}}, \mathrm{ml})$, standard deviation of the mean $(\mathrm{s} \overline{\mathrm{x}})$, coefficient of variation (per cent, C.V.), number of hours concerned (n).

\begin{tabular}{|c|c|c|c|c|c|c|c|c|c|c|}
\hline \multirow{2}{*}{$\begin{array}{c}\text { Site de réintroduction } \\
\text { Way of return }\end{array}$} & \multirow{2}{*}{$\begin{array}{l}\text { Porc } \\
\text { Pig }\end{array}$} & \multicolumn{4}{|c|}{$\begin{array}{l}2 \text { repas par jour } \\
2 \text { meals per day }\end{array}$} & \multirow{2}{*}{$\begin{array}{l}\text { Pore } \\
\text { Pig }\end{array}$} & \multicolumn{4}{|c|}{$\begin{array}{l}\text { I Repas par jour } \\
\text { I Meal per day }\end{array}$} \\
\hline & & $\bar{x}$ & $s \bar{x}$ & $\mathrm{CV}$ & $\bar{x}$ & & $\bar{x}$ & $s \bar{x}$ & $\mathrm{CV}$ & $n$ \\
\hline $\begin{array}{l}\text { Duodénum } \\
\text { Duodenum }\end{array}$ & $\begin{array}{l}\text { I } \\
2 \\
3 \\
4 \\
5\end{array}$ & $\begin{array}{l}97,79 \\
82,31 \\
91,63 \\
85,95 \\
99,57\end{array}$ & $\begin{array}{l}2,40 \\
2,04 \\
2,50 \\
2,1 \mathrm{I} \\
2,02\end{array}$ & $\begin{array}{r}26,96 \\
24,32 \\
26,8 \mathrm{I} \\
24,14 \\
31,56\end{array}$ & $\begin{array}{r}120 \\
96 \\
96 \\
96 \\
240\end{array}$ & $\begin{array}{l}6 \\
7 \\
8 \\
4 \\
5\end{array}$ & $\begin{array}{r}53,94 \\
86,38 \\
81,22 \\
67,0 \mathrm{I} \\
\mathrm{I} 12,3 \mathrm{I}\end{array}$ & $\begin{array}{l}0,97 \\
2,33 \\
2,05 \\
2,21 \\
1,98\end{array}$ & $\begin{array}{l}17,68 \\
26,40 \\
24,69 \\
28,05 \\
24,44\end{array}$ & $\begin{array}{r}96 \\
96 \\
96 \\
72 \\
192\end{array}$ \\
\hline $\begin{array}{l}\text { Cholédoque distal } \\
\text { Distal choledochus }\end{array}$ & $\begin{array}{l}\text { I } 3 \\
\text { I } 4\end{array}$ & $\begin{array}{r}\text { I03,88 } \\
90,32\end{array}$ & $\begin{array}{l}\mathrm{I}, 98 \\
\mathrm{I}, 87\end{array}$ & $\begin{array}{l}29,67 \\
32,03\end{array}$ & $\begin{array}{l}240 \\
240\end{array}$ & $\begin{array}{l}\text { I3 } \\
\text { I4 }\end{array}$ & $\begin{array}{l}93,04 \\
97,34\end{array}$ & $\begin{array}{l}6,04 \\
2,92\end{array}$ & $\begin{array}{l}71,13 \\
35,84\end{array}$ & $\begin{array}{l}\text { I } 20 \\
\text { I } 44\end{array}$ \\
\hline
\end{tabular}

\section{c) Influence des repas sur l'évolution du débit au cours des 24 heures}

L'évolution moyenne du débit biliaire, pour les porcs soumis à réintroduction intestinale et recevant 2 repas par jour, est illustrée par la figure 2 . Les différences entre porcs y sont évidentes. Globalement cependant (tous porcs et nycthémères confondus) on doit admettre que la quantité de bile émise au cours des deux heures consécutives au repas est significativement plus faible que celle qui peut être recueillie avant celui-ci dans le même temps. De surcrôit une certaine anticipation de cette réduction post prandiale, observable sur la courbe moyenne générale lors $d u$ repas de $I_{7} h$, peut être retrouvée occasionnellement (par exemple porc 5 ).

Dans le cas des porcs soumis également à réintroduction intestinale, mais ne recevant qu'un seul repas par jour, les courbes moyennes individuelles (fig. 3) 


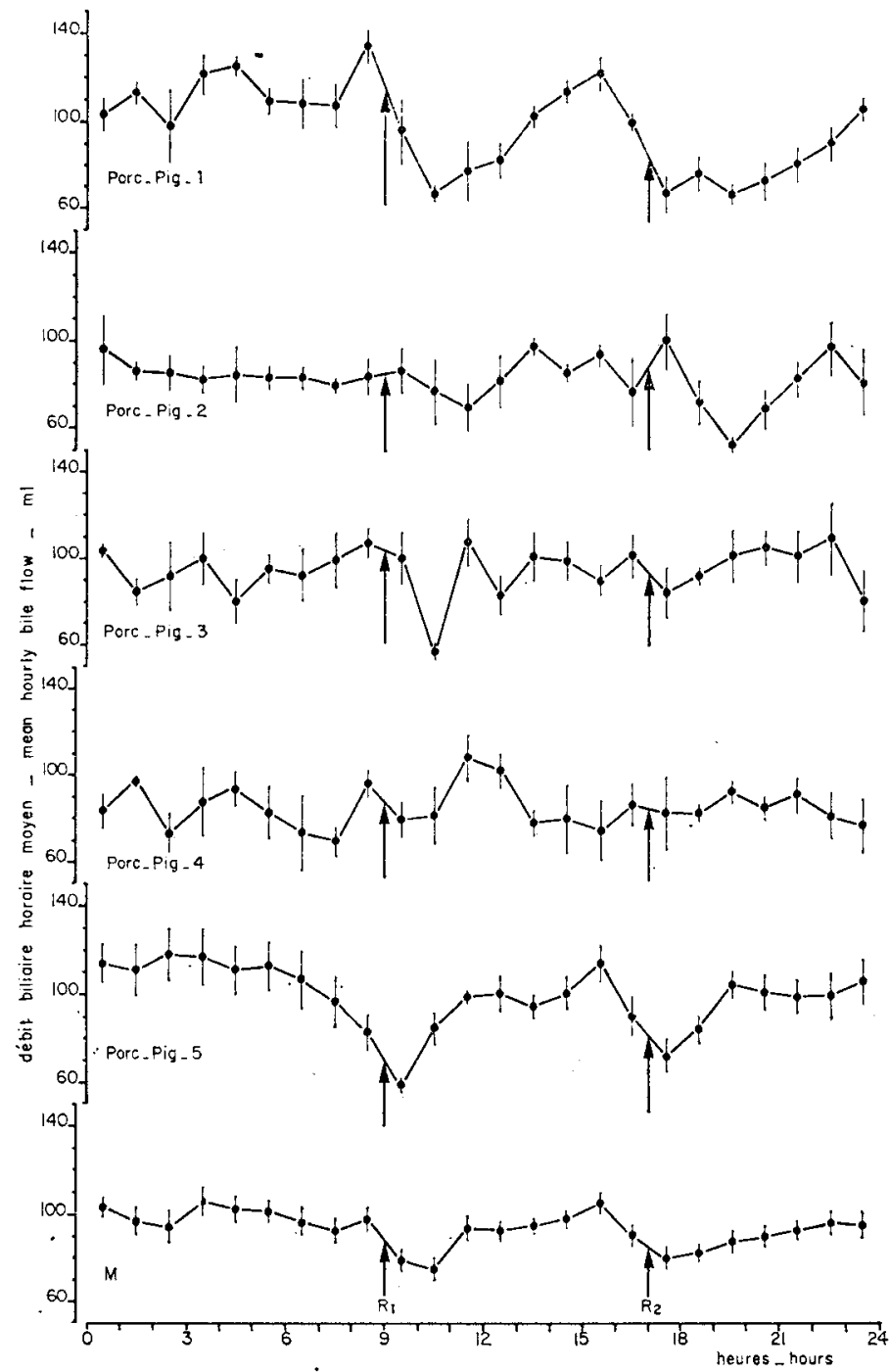

FIG. 2. - Evvolution au cours du nycthémère du débit biliaire cholédocien moyen par heure chez des porcs à sphincter d'Oddi exclu (réintroduction intestinale divecte) recevant 2 repas par jour $\grave{a} 9 h$ et $I 7 h(R I$ et R2): courbes moyennes, avec écarts-types de la valeur horaive moyenne, basées sur: Porc I: 5 jours - Porc 2: 4 jours - Porc 3: 4 jours - Porc $4: 4$ jours - Porc 5 : ro jours - M: Moyenne générale des 27 nycthémères de 5 porcs.

Mean hourly bile flow from choledochus fistulated pigs, bile being returned divectly into the duodenum, throughout the $24 \mathrm{~h}$ period. $R I$ and $R z: 2$ meals per day at 9 a.m. and 5 ip.m. $\rightarrow$ Mean curves with standard deviation of the hourly mean flow: Pig I:5 days - Pig $2: 4$ days - Pig 3 : 4. days - Pig 4:4 days - Pig 5: xo days. M: gross mean of 27 days from the 5 pigs.

offrent une augmentation au cours des 3 heures post prandiales chez les porcs 5 et 8 , mais une diminution chez les porcs 4 et 7 . Il en résulte le relatif nivellement de la courbe moyenne générale. On notera cependant que sur cette dernière courbe l'heure de distribution du repas unique coïncide avec le niveau le plus bas du débit biliaire moyen horaire au cours du nycthémère. 


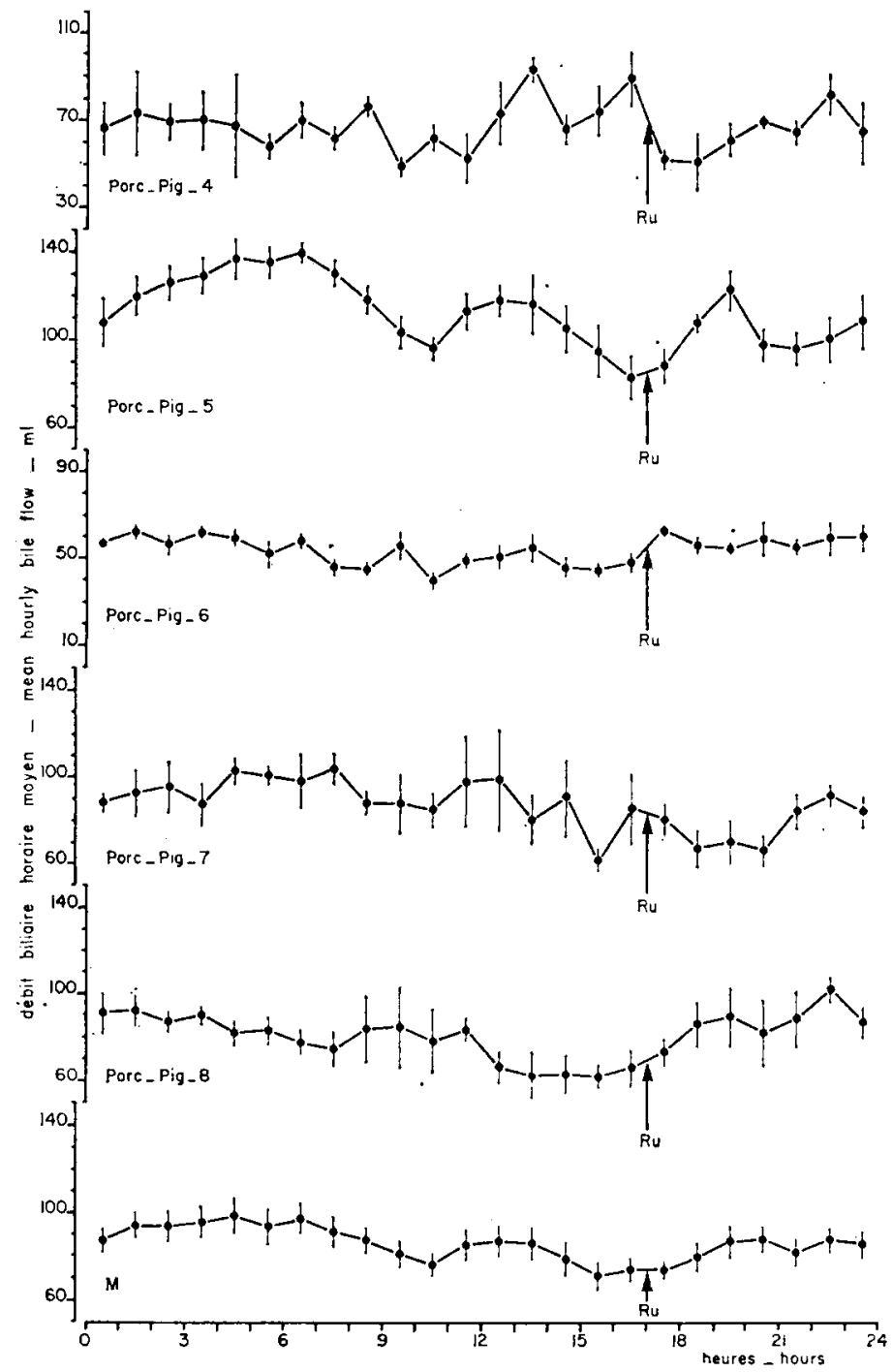

FIG. 3. - Évolution au cours du nycthémère du débit biliaire cholédocien moyen par heure chez des porcs à sphincter d'Oddi exclu (réintroduction intestinale directe) recevant un seul repas quotidien à I $h(R u)$; courbes moyennes, avec écarts-types de la valeur horaire moyenne, basées sur; Porc $4: 3$ jours — Porc $5: 8$ jours - Porc 6: 4 jours - Porc $7: 4$ jours - Porc 8: 4 jours $M$ : moyenne générale des 24 nycthémères de 5 porcs.

Mean hourly bile flow from choledochus fistulated pigs, bile being veturned divectly into the duodenum, throughout the $24 \mathrm{~h}$ period. Ru: only I meal per day at 5 p.m. Mean curves with standard deviation of the hourly mean flow: Pig $4: 3$ days - Pig 5:8 days-Pig 6:4 days - Pig $7:$ 4 days - Pig 8:4 days - $M:$ gross mean of 24 days from the 5 pigs.

Bien qu'il s'agisse d'une courbe établie sur la base de plusieurs nycthémères au même rythme de distribution unique d'aliment, chez des animaux accoutumés au préalable à ce rythme, on observe également une dépression transitoire du débit 
entre $9 \mathrm{~h}$ et II $\mathrm{h}$. Celle-ci peut être retrouvée sur la courbe moyenne individuelle du potc 5 sans que l'on puisse savoir s'il s'agit d'un phénomène accidentel ou d'une rémanence d'effet du traditionnel repas du matin.

Cependant le ralentissement du débit biliaire horaire moyen au cours des 2 heures consécutives à l'un ou l'autre des repas, lors de double distribution, semble bien être un phénomène associé au repas et non une coïncidence.

En effet, cette réduction du niveau sécrétoire peut être déplacée dans le nycthémère, sans habituation préalable, par simple modification des horaires de distribution (fig. 4). Ire caractère "instantané » de cette réponse s'oppose donc à l'hypothèse de rémanence possible émise précédemment.

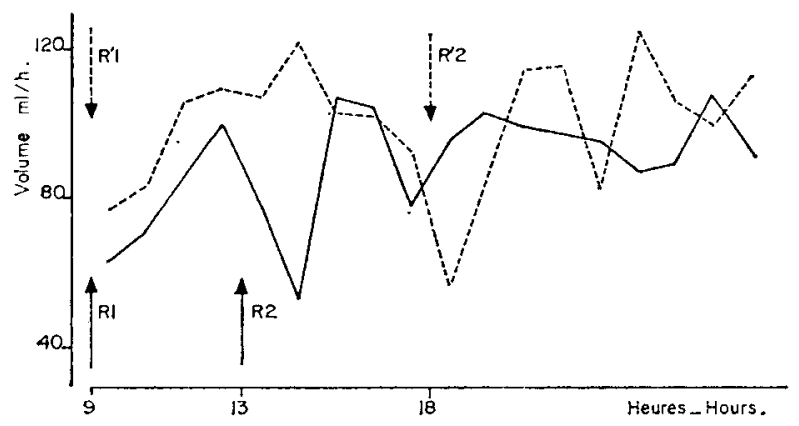

FIG. 4. - Déplacement de la dépression du débit biliaire horaive moyen au cours des 2 heures post. prandiales par modification des horaires de distribution des repas, sans habituation préalablePorc II, à sphincter d'Oddi exclu: 2 repas de $800 \mathrm{~g}$ d'aliment sec à $9 h$ et $x_{3} h(R x-R \boldsymbol{z})$, ou $\grave{a}$ $9 h$ et $I 8$ h $\left(R^{\prime} I-R^{\prime} 2\right)$.

Displacement in the $24 \mathrm{~h}$ period of the decreased mean hourly bile flow during the first 2 hours after the meal, by changing the feeding time without previous habituation. Pig II, with duodenal restitution of bile (Oddi's sphincter excluded) : 2 meals of $800 \mathrm{~g}$ of flour each, at 9 a.m. and I p.m. $(R I-R z)$ or at 9 a.m. and 6 p.m. (R'I-R'2).

\section{d) Effets immédiats du repas et vidange vésiculaire}

L'analyse comparative des volumes de bile émis au cours des $30 \mathrm{mn}$ précédant la distribution et des $30 \mathrm{mn}$ consécutives au début du repas, conduit à enregistrer des résultats divergents : le débit moyen par $5 \mathrm{mn}$ durant ces deux périodes (tabl. 3 ) s'avère significativement réduit après 1'un ou l'autre des 2 repas mais significativement accru après un repas quotidien unique, chez un même porc soumis à réintroduction intestinale.

En dépit de cette image caractéristique (fig. 5 - hémigraphique gauche) certains enregistrements peuvent conduire à reconnaitre l'existence au moment du repas d'un accroissement transitoire de l'écoulement de bile, avec anticipation témoignant du conditionnement de pores habitués à un horaire fixe. On peut aussi observer un véritable pic sécrétoire; mais ce phénomène particulier succède à une période d'inhibition totale du débit durant plusieurs minutes et se rencontre occasionnellement chez un animal manifestant un état d'excitation considérable (usuel en distribution quotidienne unique). 

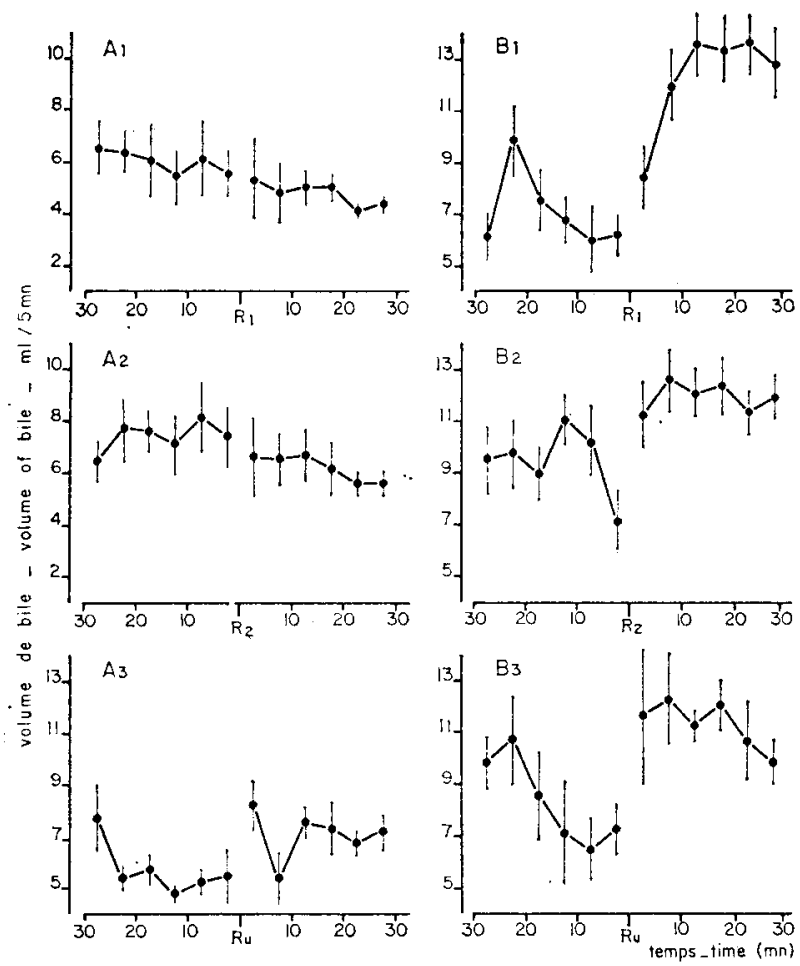

FIG. 5. - Évolution du débit biliaire cholédocien moyen par fraction de $5 \mathrm{mn}$ au cours des $30 \mathrm{mn}$ qui précèdent et des 30 mn qui suivent la distribution d'aliment. Demi-graphique gauche: chez des porcs auxquels la bile est restituée directement dans le duodénum $(A)$; demi-graphique droit: chez des porcs auxquels la bile est restituée par perfusion du bas cholédoque, irrigant le sphincter d'Oddi $(B)$.

- Repas à $9 \mathrm{~h}$ lors de double distribution quotidienne :

- $A r$, porc 5 , moyenne de 9 épreuves,

- $B I$, porc I2, moyenne de ro épreuves.

- Repas à $\mathrm{I} 7 \mathrm{~h}$ lors de double distribution quotidienne :

- $A 2$, porc 5 , moyenne de ro épreuves,

- $B 2$, porc I2, moyenne de II épreuves.

- Repas à 17 h lors de distribution quotidienne unique :

- $A 3$, porc 5 , moyenne de 8 épreuves,

- $B_{3}$, porc I4, moyenne de 6 épreuves.

Mean bile flow per $5 \mathrm{mn}$ during $30 \mathrm{mn}$ before and $30 \mathrm{mn}$ after feeding in choledochus fistulated pigs. On the left: duodenal restitution of bile $(A)$; on the right: bile being returned into the distal choledochus, thus flowing through the Oddi's sphincter $(B)$.

- Meal at 9 a.m. in the case of 2 meals per day :

- $A I$, pig 5, mean of 9 tests,

- $B I$, pig I2, mean of Io tests.

- Meal at 5 p.m. in the case of 2 meals per day :

- $A 2$, pig 5 , mean of ro tests,

- B2, pig I2, mean of $\mathrm{Ir}$ tests.

- Meal at 5 p.m. in the case of only $I$ daily meal :

- $A 3$, pig 5 , mean of 8 tests

- $B_{3}$, pig I 4 , mean of 6 tests. 
TABLEAU 3

Débit moyen de la bile ( $\mathrm{ml} / 5 \mathrm{mn}$ ) au cours des $30 \mathrm{mn}$ avant et des $30 \mathrm{mn}$ après le début du repas selon le mode de restitution de la bile à l'animal, et le numéro du repas d'épreuve considéré ( $R I$ et R2 lors de double distribution par jour - Ru lors de distribution quotidienne unique).

$n=$ nombre de repas d'épreuve.

$\bar{x}=$ moyenne, $s \bar{x}=$ écart-type de la moyenne.

Signification statistique : test $t$ de Student.

Mean bile flow ( $\mathrm{ml}$ per $5 \mathrm{mn}$ ) during $30 \mathrm{mn}$ before and $30 \mathrm{mn}$ after the meal time, according to the way of bile restitution to the pig and to the number of the meal (RI and R2 in the case of 2 meals per day — Ru: only one meal per day).

$n=$ number of test-rieals.

$\bar{x}=$ mean $s \bar{x}=$ standard deviation of the mean.

Statistical significance : Student $t$ test.

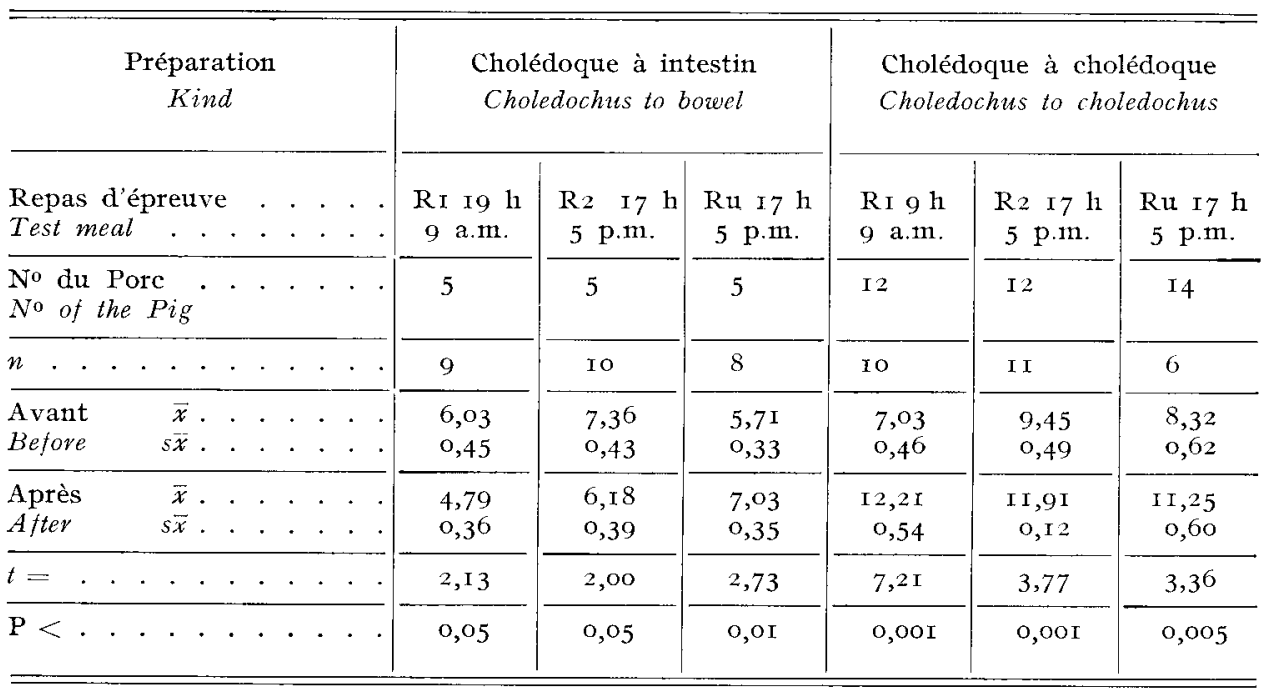

\section{II. - Débit biliaire cholédocien lors de perfusion oddienne}

a) Aspects quantitatifs.

Le volume total de bile collecté en $24 \mathrm{~h}$, chez les pores dont le trajet sphinctérien de 1'Oddi est irrigué à débit constant par la bile réintroduite dans le cholédoque terminal, est du même ordre de grandeur que lors de réintroduction directe dans l'intestin. Cependant, la comparaison des pools de données, pour chacune des préparations et selon le nombre de distributions alimentaires, montre (tabl. 4) que, indépendamment du nombre de repas, la voie de réintroduction de la bile est responsable d'une modification. En effet, le débit biliaire par $24 \mathrm{~h}$ est en moyenne plus élevé $(t=3,00-\mathrm{P}<0,0 \mathrm{I})$ chez les porcs à réintroduction sus-oddienne $(2408,7$ $\pm 45,0 \mathrm{ml}$ sur 42 nycthémères) que chez les porcs à réintroduction intestinale directe ( 2 I 43,4 $\pm 70,9 \mathrm{ml}$ sur $5 \mathrm{I}$ nycthémères).

Les quantités totales moyennes recueillies en $24 \mathrm{~h}$, pour chaque porc soumis 


\section{TABLEAU 4}

Quantités totales moyennes (mi) de bile collectée en 24 h au niveau du cholédoque chez le porc selon qu'elle est restituée à l'animal directement dans l'intestin ou dans le bas cholédoque, et selon le nombre de repas quotidiens.

Mean total volume $(\mathrm{ml})$ of bile collected from the choledochus during $a 24$ period in the Pig, according to the way used to return the bile to the animal (into the duodenum or the distal choledochus), and to the number of daily meals.

\begin{tabular}{|c|c|c|c|c|}
\hline $\begin{array}{l}\text { Mode de circulation } \\
\text { de la bile } \\
\text { Mode of bile } \\
\text { sircilation }\end{array}$ & $\begin{array}{c}\text { Nombre } \\
\text { de nycthémères } \\
\text { Number } \\
\text { of } 24 \text { periods }\end{array}$ & $\begin{array}{l}\text { Repas /jour } \\
\text { Meal/day }\end{array}$ & $\begin{array}{l}\text { Volume total } \\
\text { moyen de bile } / \\
\text { Mean total volume } \\
\text { of bile } / 24 h\end{array}$ & $\begin{array}{l}\text { test } t \text { de Student } \\
\text { Student } \mathrm{t} \text { test }\end{array}$ \\
\hline $\begin{array}{l}\text { Cholédoque à l'in- } \\
\text { testin . . . } \cdot \text {. } \\
\text { Choledochus to the } \\
\text { bowel . . . . . }\end{array}$ & $\begin{array}{c}27 \text { (5 porcs) } \\
(5 \text { pigs })\end{array}$ & 2 & $\begin{array}{l}224^{T, 4} \\
\pm 70,2\end{array}$ & \multirow{2}{*}{$\begin{array}{l}t=2,33 \\
\mathbf{P}<0,05\end{array}$} \\
\hline $\begin{array}{l}\text { Choledoque au cho- } \\
\text { lédoque. } \\
\text { Choledochus to chole } \\
\text { dochus. }\end{array}$ & $\begin{array}{c}3 \text { (3 pores) } \\
\text { (3 pigs) }\end{array}$ & 2 & $\begin{array}{l}244^{8,4} \\
\pm 5^{6, I}\end{array}$ & \\
\hline $\begin{array}{l}\text { Cholédoque à l'in- } \\
\text { testin . . . } \\
\text { Choledochus to the } \\
\text { bowel . . . . . }\end{array}$ & $\begin{array}{c}23 \text { (5 pores) } \\
(5 \text { pigs })\end{array}$ & I & $\begin{array}{l}2072,1 \\
\pm 125,7\end{array}$ & \multirow{2}{*}{$\begin{array}{l}t=\mathrm{I}, \mathrm{I9} \\
\text { N.S. }\end{array}$} \\
\hline $\begin{array}{l}\text { Cholédoque au cho- } \\
\text { lédoque. . . - } \\
\text { Choledochus to cho- } \\
\text { ledochus . . . }\end{array}$ & $\begin{array}{l}\text { I I }(2 \text { pores }) \\
\text { (2 pigs) }\end{array}$ & I & $\begin{array}{l}2296,3 \\
\pm 5^{8,5}\end{array}$ & \\
\hline
\end{tabular}

à une réintroduction sus-oddienne, sont indiquées dans le tableau 5 . La variabilité observée dans les deux groupes de données (3I nycthémères de 3 porcs/II nycthémères de 2 porcs) est de $\mathrm{I} 2,8 \mathrm{p}$. cent ( 2 repas par jour) et de $8,4 \mathrm{p}$. cent ( $\mathrm{I}$ repas par jour) soit des coefficients de variation nettement inférieurs à ceux calculés pour les porcs soumis à réintroduction intestinale. Pourtant, le volume total moyen pour 24 h est significativement plus faible $(\mathrm{P}<0$,OI) chez le porc I4 que chez les 2 autres animaux à réintroduction sus-oddienne recevant également 2 repas par jour.

Les différences entre simple et double distribution d'aliment, testées chez les porcs I 3 et 14 , montrent une réduction significative $(\mathrm{P}<0,02)$ du volume de bile par $24 \mathrm{~h}$ lors de repas unique pour le porc $\mathrm{I}_{3}$, mais à l'inverse une augmentation non significative pour le porc I4. Globalement, les volumes moyens obtenus en $24 \mathrm{~h}$, pour $3 \mathrm{I}$ nycthémères de 3 porcs recevant 2 repas et II nycthémères de 2 porcs recevant un seul repas, ne diffèrent pas significativement.

L'influence du niveau d'ingestion, testée dans les conditions de double distribution alimentaire pour des niveaux de 500 à I $600 \mathrm{~g}$ ou 500 à $2000 \mathrm{~g}$ d'aliment sec par jour, n'est en aucun cas significative. 


\section{TABLEAU 5}

Quantités totales moyennes (ml) de bile collectée en 24 h chez des porcs à fistulation cholédocienne et réintroduction dans le bas cholédoque (irrigation du sphincter d'Oddi). Les distributions d'aliment interviennent systématiquement à 9 h et $I_{7} h$ ou à 17 h lors de repas unique.

Mean total volume (m1) of bile collected during a 24 h period in pigs fitted with a fistula of the choledochus and having bile returned into the distal choledochus, thus flowing through the Oddi's sphincter. Meal time $=9$ a.m and 5 p.m, or 5 p.m only.

\begin{tabular}{|c|c|c|c|c|}
\hline $\begin{array}{l}\text { Porc } \\
\text { Pig }\end{array}$ & $\begin{array}{c}\text { Nombre } \\
\text { de nycthémères } \\
\text { Number } \\
\text { of } 24 \mathrm{~h} \text { periods }\end{array}$ & $\begin{array}{l}\text { Repas/jour } \\
\text { Meal /day }\end{array}$ & $\begin{array}{l}\text { Consommation } \\
\text { moyenne/jour } \\
\text { Mean daily } \\
\text { intake/g }\end{array}$ & $\begin{array}{c}\text { Volume total } \\
\text { moyen de bile } / 24 \mathrm{~h} \\
\text { Mean total volume } \\
\text { of bile } / 24 \mathrm{~h}\end{array}$ \\
\hline . . . . . . . & I I & 2 & r 300 & $2632,9 \pm 96,0$ \\
\hline$. \cdot . \cdot . \cdot \cdot \cdot$ & Io & 2 & I 800 & ${ }^{2} 5^{\mathrm{I}} 3, \mathrm{O} \pm 34,5$ \\
\hline $14 \ldots \ldots$ & Io & 2 & I 700 & $2 \mathrm{I} 8 \mathrm{I}, 4 \pm 86,9$ \\
\hline Moyenne-Mean. . . . & $n=3 \mathbf{I}$ & 2 & I 630 & $244^{8,4} \pm 5^{6,1}$ \\
\hline$\cdot . \cdot . \cdot \cdot \cdot$ & 5 & I & I $74^{\circ}$ & $2253,0 \pm 114,7$ \\
\hline $\mathbf{I} 4 \ldots \ldots$ & 6 & $\mathbf{I}$ & 2000 & $233^{2 \cdot 3} \pm 56,5$ \\
\hline Moyenne-Mean . . . & $n=\mathrm{I} \mathrm{I}$ & I & I $880^{\circ}$ & $2296,3 \pm 5^{8,5}$ \\
\hline
\end{tabular}

\section{b) Variabilité du débit biliaire au cours des 24 heures}

L'irrégularité du débit de bile par unité de temps de $5 \mathrm{mn}$ est, là encore, considérable. Elle est responsable de l'importance de la variabilité du débit au cours d'une même tranche horaire du nycthémère. On enregistre ainsi chez ces porcs à réintroduction sus-oddienne des coefficients de variation du débit au cours d'une même heure compris, selon l'heure considérée, entre :

- 19,5 et 44,I p. cent sur la base de 20 nycthémères ( 2 repas/jour),

- r5,5 et 9I,7 p. cent sur la base de II nycthémères ( $\mathrm{I}$ repas/jour).

La variabilité globale du volume de bile émis en moyenne par heure, calculée porc par porc, a été indiquée avec les valeurs moyennes dans le tableau 2 , comparativement à celle calculée pour les animaux à réintroduction intestinale.

\section{c) Influence des repas sur l'évolution du débit au cours des $24 \mathrm{~h}$}

L'évolution du débit biliaire horaire moyen tout au long du nycthémère chez les porcs soumis à réintroduction sus-oddienne est illustrée par la figure 6 pour les deux fréquences de distribution alimentaire. Chez les animaux ingérant 2 repas par jour, la prise de nourriture paraît suivie d'une augmentation de la production de bile durant une heure; le niveau sécrétoire décroît ensuite $\left(2^{\mathrm{e}}\right.$ heure post prandiale) de $I_{5}$ à $30 \mathrm{ml} / \mathrm{h}$. Par ailleurs, la courbe de débit horaire moyen passe par un maximum alentour de $\mathrm{I}_{4} \mathrm{l}$.

Les pores à réintroduction sus-odđienne n'ingérant qu'un seul repas par jour 


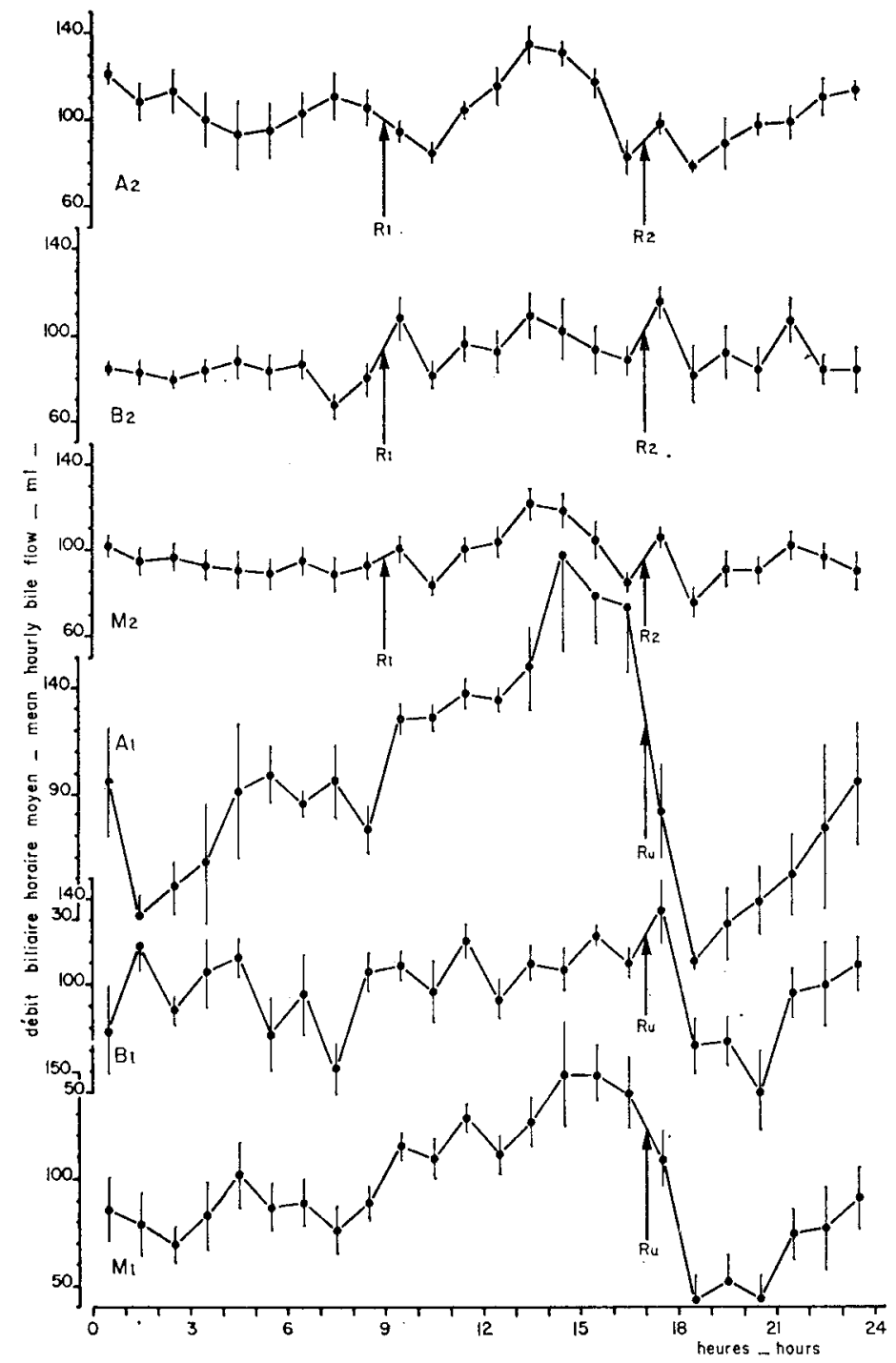

FIG. 6. - Évolution au cours du nycthémève du débit biliaire cholédocien moyen par heure chez des porcs dont le sphincter d'Oddi est irrigué par la bile réintroduite à débit constant dans le cholédoque distal. Courbes moyennes, avec écarts-types de la valeur hovaire moyenne.

$A \mathrm{I}, B \mathrm{I}, M_{\mathrm{I}}$ : lors de distribution quotidienne unique d'aliment à $\mathrm{I}_{7} \mathrm{~h}(\mathrm{Ru})$.

$A 2, B 2, M 2$ : lors de double distribution quotidienne d'aliment à $9 \mathrm{~h}$ (RI) et $\mathrm{I}_{7} \mathrm{~h}(\mathrm{R} 2)$. $A 2$, porc I3, Io jours - $A I$, porc I3, 5 jours.

$B 2$, pore $\mathrm{I}_{4}$, Io jours $-B x$, pore $x_{4}, 6$ jours.

$M 2$, moyenne générale des 20 nycthémères de 2 porcs.

$M I$, moyenne générale des I I nycthémères de 2 porcs.

Mean hourly bile flow from choledochus fistulated pigs, throughout the 24 h period, bile being returned into the distal choledochus at a constant rate, thus flowing through the Oddi's sphincter. Mean curves with standard deviation of the hourly mean flow.

$A I, B I, M_{I}$ : in the case of only I meal per day at 5 p.m. (Ru).

$A 2, B 2, M 2:$ in the case of 2 meals per day at 9 a.m. (R 1 ) and 5 p.m. (R2).

$A 2$, pig 13 , ro days $-A I$, pig 13,5 days.

$B 2$, pig I 4 , Io days $-B I$, pig I 4,6 days.

M2, gross mean of 20 days from 2 pigs.

$M I$, gross mean of I I days from 2 pigs. 
fournissent une courbe très remarquable avec un maximum (I4-I5 h) et un minimum entre I 8 et $2 \mathrm{I} \mathrm{h}$. La décroissance brutale du débit horaire moyen fait suite à l'ingestion de l'unique repas quotidien.

\section{d) Effets immédiats du repas et vidange vésiculaire}

La comparaison des volumes de bile émis au cours des $30 \mathrm{mn}$ précédant le repas et des 30 mn qui lui font suite, démontre (tabl. 3) pour les porcs à réintroduction sus-oddienne, quels que soient le repas considéré et la fréquence de distribution un accroissement du débit biliaire de 30 à $7^{\circ} \mathrm{p}$. cent. La représentation graphique (fig. 5) des volumes émis par unité de temps de $5 \mathrm{mn}$ met en évidence le caractère immédiat de cette augmentation du débit à partir du début du repas. L'effet se prolonge au-delà de l'achèvement de celui-ci (durée d'ingestion de l'ordre de 7 à $9 \mathrm{mn}$ selon la quantité consommée).

\section{Discussion et conclusions}

D'un point de vue strictement quantitatif (volume total de bile produite en $24 \mathrm{~h}$ ) quelques faits peuvent être retenus : globalement, ce volume est plus important lorsque la bile est restituée à l'animal par perfusion à débit constant du bas cholédoque, irrigant ainsi le sphincter d'Oddi, que lorsqu'elle est réintroduite dans les mêmes conditions de débit directement dans la lumière intestinale.Cette quantité quotidienne de bile n'est pas systématiquement affectée par le rythme de distribution alimentaire ( $\mathbf{I}$ ou 2 repas/jour). Mais il est bien certain que 1'affirmation, reprise par DAVENPORT (I968) selon laquelle le Porc (comme la Chèvre, le Mouton et la Vache) possède une vésicule biliaire à faible pouvoir de concentration, mais que son foie sécrète des quantités relativement faibles de bile, ne peut être acceptée face à des sécrétions supérieures à 2 litres par jour chez des porcs de poids vif compris entre 57 et $63 \mathrm{~kg}$ ( 800 à I $000 \mathrm{ml}$ chez 1 'homme). Par ailleurs, les résultats de EGGER et al. (I974) semblent confirmer pour cette espèce une concentration notable dans la vésicule. La différence quantitative de bile sécrétée en $24 \mathrm{~h}$ selon qu'elle est restituée à l'animal par voie cholédocienne ou par voie duodénale, pourrait impliquer un certain rôle des formations réceptives décrites par DeKhKanov (1974) dans le contrôle de la cholérèse.

La variabilité des débits biliaires enregistrés est dans l'ensemble extrêmement élevée. Le fait peut paraître normal pour des débits par $5 \mathrm{mn}$, mais les volumes émis par heure sont, pour une même période du cycle de $24 \mathrm{~h}$, fort variables d'un jour à l'autre. Cet effet du jour de collecte est doublé d'un effet animal, très sensible pour les volumes de bile produite en 24 h. La cas du porc 6 en est un exempletype dans la mesure où rien dans l'état clinique de l'animal ne permet de suspecter la validité des données, et où il n'existe généralement aucune relation linéaire entre le volume quotidien de bile et la quantité d'aliment ingérée par jour.

Le tableau ainsi obtenu de la production de bile chez le Porc doit donc être accepté comme relativement fluctuant dans la mesure où rien n'autorise à renier telle ou telle mesure; cette constatation diffère de la relative constance de la production de bile par $24 \mathrm{~h}$, pour un régime alimentaire standard, autrefois décrite par Scнміт t et al. (1939). Il faut toutefois souligner que la variabilité des volumes par 24 h est beaucoup plus faible chez les porcs soumis à réintroduction sus-oddienne ( 12,8 p. cent - 2 repas/jour; 8,4 p. cent - I repas/jour) que chez les pores à réintroduction intestinale directe ( $\mathrm{I} 6,3 \mathrm{p}$. cent -2 repas /jour; $29, \mathrm{I}$ p. cent - I repas / jour). 
La répartition dans le nycthémère des volumes collectés suggère l'existence d'un cycle, reconnue dès I 890 par DASTRE. Cet auteur décrit un cycle à 2 maxima (à 9 et $22 \mathrm{~h}$ ). L'existence d'un cycle a été confirmée peu après chez l'homme : sécrétion maximum de jour et minimum la nuit (Koster, Shapiro et LERnER, I936; JoSEPHSON, I94I). De plus, FORSGREN (I930) précise que le rythme nycthéméral de la sécrétion biliaire n'a pas de rapport direct ou constant avec l'alimentation.

Chez le Porc, nous n'observons aucune relation régulière entre la quantité de bile produite en $24 \mathrm{~h}$ et le niveau d'ingestion alimentaire, sans préjuger de la possibilité de changements quantitatifs associés à des modifications de la composition ou nature de l'aliment. Nous constatons aussi une influence certaine des repas sur l'évolution du débit biliaire au cours des 24 hettres. Toutefois l'ampleur et la variété des phénomènes observés ne permet nullement de supposer la forme possible d'un éventuel rythme nycthéméral authentique, en 1'absence d'enregistrement de la sécrétion basale chez des porcs à jeûn.

Par ailleurs, le décours de la sécrétion biliaire au long des $24 \mathrm{~h}$ ne permet pas, chez le porc normalement alimenté, d'admettre l'affirmation usuelle que l'état de digestion renforce le flux biliaire hépatique. Nous avons en effet observé lors de réintroduction intestinale, une réduction significative du débit au cours des 2 heures postprandiales ( 2 repas par jour) et la coïncidence du débit horaire moyen le plus faible avec l'heure de distribution du repas unique. Lors de réintroduction sus-oddienne, et en particulier lors de repas unique, on note encore que le maximum précède 1'heure du repas alors que le minimum lui fait suite. Cette influence, apparemment dépressive du débit biliaire, exercée par les repas, est à la fois surprenante et trop prolongée pour que 1'on puisse envisager le problème sous l'angle d'une stimulation tardive de la cholérèse (après 3 ou $4 \mathrm{~h}$ ) et non de son inhibition précoce ( 2 premières heures). Compte tenu de ce que l'on sait de la précocité de l'évacuation gastrique chez le Porc et de l'importance de ce phénomène aut cours des 2 premières heures (AufFray, Mar'Tine'T et RERAT, I967; LAPLACE et TomasSONE, I970), il est évident que les processus de digestion sont largement engagés alors même que la sécrétion biliaire est réduite.

Une autre hypothèse peut être considérée pour expliquer ces coïncidences si l'on tient compte du fait que la restitution de bile à l'animal est effectuée à débit constant tout au long du nycthémère. Cette condition très particulière implique qu'à 1'arrivée dans le duodénum d'une quantité importante de matériaux alimentaires ne peut correspondre de toute façon un afflux biliaire important. De ce fait la quantité de sels biliaires présents dans la lumière digestive se trouve, en valeur relative à la masse de digesta, beaucoup plus faible à la suite du repas. La proportion de sels biliaires impliqués dans le processus de digestion étant accrue, celle qui peut très rapidement faire l'objet d'un recyclage entéro-hépatique se trouve réduite. La dépression de la sécrétion biliaire à la suite des repas serait, dans cette hypothèse, liée à une diminution quantitative de l'effet cholérétique des sels biliaires Une telle hypothèse concorderait avec la conclusion de EGGER et al. (I974) selon lesquels la formation de la bile chez le Porc dépend principalement de l'excrétion des sels biliaires. Dans ce sens on pourrait aussi attribuer 1'anticipation de la réduction de sécrétion sur le repas à l'évacuation gastrique résiduelle du repas précédent (" fonds d'estomac ") souvent observée chez l'animal accoutumé à un horaire et qui attend donc la distribution d'aliment. Compte tenu de ces éléments, seule une étude directe des quantités de sels biliaires présentes dans le sang portal permettra de juger de la validité de cette hypothèse. Toute rythmicité apparente liée aux repas ne serait alors que la stricte conséquence du mode de réintroduction 
à débit constant ici utilisé. La recherche d'un rythme nycthéméral véritable ne peut donc être envisagée que dans des conditions de restitution de la bile rigoureusement calquées sur le débit cholédocien.

Nous avons antérieurement observé la réalité (LAPLACE, I976b) de 1'évacuation vésiculaire post prandiale par enregistrement simultané du débit biliaire physiologique à l'ampoule de Vater et de l'activité électromyographique des voies biliaires. Il apparaît cependant que, lors d'élévation du débit biliaire dans les instants du repas, celle-ci ne porte que sur un volume assez faible par rapport à la capacité de la vésicule de porc. En effet, les volumes de bile vésiculaire constatés post-mortem chez 16 porcs de roo $\mathrm{kg}$ pris au hasard sur la chaine d'abattage fournissent 2 populations de données : pour 8 pores le volumie moyen est de 36 , I 2 $\pm 4,30 \mathrm{ml}$; pour les 8 autres, i1 est de $96,75 \pm 6,40 \mathrm{ml}$. La confrontation de ces deux ordres d'observations et l'absence de témoin électrique de la contractilité vésiculaire (LAPLACE, I976a) suggèrent que cette dernière est chez le Porc relativement modeste. Le Porc constituerait sur ce plan un intermédiaire entre carnivores à vésicule très contractile et herbivores à vésicule infonctionnelle lorsqu'elle existe anatomiquement.

Si tentante que soit 1'hypothèse émise ci-dessus, il paraît souhaitable de réexaminer ce phénomène dans les conditions d'une réintroduction calquée sur l'émission de bile, ainsi que d'étudier la réactivité de la vésicule de porc à la cholécystokinine (C.C.K.). Bien que dans nos conditions le débit biliaire hépatique soit au plus bas lors des repas, l'effet cholagogue de la prise de nourriture reste très modéré. Mais compte tenu des travaux de BANFIELD (r975) éclairant le mécanisme d'inhibition de la libération de cholécystokinine, il est également possible que l'apport continu dans la lumière duodénale d'un débit constant de bile constitue une condition défavorable à une libération normale de cette hormone. De telles interférences pourraient être directes ou indirectes dans la mesure où des influences de la sécrétion pancréatique sur la sécrétion biliaire (GRENIER et DELAGE, Ig60) ou réciproquement de celle-ci sur la sécrétion pancréatique (VOGEL, GROT'T et KuRTEN, Ig66) ont été signalées. Mais les controverses dans ce domaine ne permettent pas d'évaluer la nature des phénomènes en cause.

Quoi qu'il en soit, et si modéré que soit 1'effet cholagogue de la prise de nourriture, il existe bien une différence spectaculaire illustrée par la figure 5, entre porcs à réintroduction intestinale et porcs à réintroduction sus-oddienne. Cette particularité doit être envisagée en relation avec le rôle possible des formations réceptrices décrites par DEKHKANOV (I974). Celles-ci sont susceptibles de constituer le point de départ de réflexes entre la vésicule biliaire et le sphincter d'Oddi. Il est donc plausible que l'irrigation du bas cholédoque et du sphincter d'Oddi par la bile réintroduite contribue à l'entretien de tels mécanismes nerveux. On sait par ailleurs que ceux-ci peuvent être abolis par la section du canal cholédoque et des plexus nerveux qui le couvrent (Hopton, I973). La mise en place des tubes dans le canal cholédoque est nécessairement responsable de leur interruption partielle, ce qui pourrait expliquer la modicité des évacuations vésiculaires postprandiales observées.

La question se trouve posée de l'importance relative des contrôles ner. vettx et humoral qui s'exercent sur la vésicule biliaire, dans une situation excluant une simple intervention de la diminution quantitative de l'effet cholérétique des sels biliaires. Il y a en effet convergence des observations faites sur les débits par $5 \mathrm{mn}$ en période péri-prandiale et des constatations faites sur l'évolution du débit horaire en période post-prandiale. Néanmoins le caractère immédiat de l'augmentation du débit lors du début du repas chez les seuls porcs à réintroduction sus- 
oddienne suggère l'intervention, dans le contrôle de la fonction vésiculaire, du mécanisme réflexe postulé dans le cadre de la coordination des activités motrices antropyloriques, duodénales et oddiennes (LAPLACE, I976 a et $b$ ).

En conclusion: cette analyse des effets des repas sur le débit biliaire cholédocien selon le site de réintroduction de la bile, fournit un certain nombre d'éléments favorables à l'hypothèse d'un rôle des formations réceptrices localisées au niveau du traiet sphinctérien à l'égard du fonctionnement de la vésicule biliaire d'une part et du contrôle de la cholérèse d'autre part.

Accepté pour publication en juin 1977.

\title{
Summary
}

\author{
Bile excretion in the pig. Influence of the meals and possible function \\ of Oddi's sphincter receptors to control the choledochus flow rate
}

The flow rate of bile secretion, collected by a choledochus fistula, was measured in 44 Large White pigs weighing between 57 and $63 \mathrm{~kg}$. The bile was restituted at a constant rate during the $24 \mathrm{hr}$ cycle either through the intestinal junction of the choledochus duct, thus irrigating the Oddi's sphincter, or directly into the duodenal lumen. The variability of the bile flow from one day to the other in the same animal, and from one animal to another seemed to be rather large, but a general relationship with the feed intake level was not established. The meals seemed to be responsible for a sort of rhythm in the hourly bile flow during the 24 hr cycle. The bile flow was generally subjected to a more or less early and prolonged reduction at the moment of feeding or thereafter. This fact might be correlated with the constancy in the rate of bile restitution to the animal. The short lasting variations in the flow rate during the immediate periprandial period suggest that the gall bladder emptying only occured when the Oddi's sphincter was irrigated by the bile restituted to the animal. However, the volume emptied was rather small.

The total amounts of bile produced (about 21 within $24 \mathrm{hr}$ ) were significantly higher after reintroduction of the bile above the Oddi's sphincter. These facts are in favour of the hypothesis according to which the receptors located in the Oddi's sphincter play a part in the function of the gall bladder and in the regulation of the choleresis.

\section{Références bibliographiques}

Auffray P., Martinet J., RERAT A., I967. Quelques aspects du transit gastro-intestinal chez le Porc. Ann. Biol. anim. Bioch. Biophys., 7, 26r-279.

BANFIELD W. J., x975. Physiology of the gallbladder. Gastroenterology, 69, 770-777.

DASTRE A., I89o. Variations diurnes de la sécrétion biliaire. Arch. physiol. norm. pathol., 22 , $800-809$.

DAvenPoRT H. W., r968. Physiologie de l'appareil digestif. Chap. II : Sécrétion de la bile, p. I56, I vol., Masson Ed., Paris.

Deknkanov T. D., I974. On the receptors of bile excreting pathways (en Russe). Arkh. Anat. Gistol. Embriol., 67, x 10-1 13 .

EGger G., Kutz K., STrebel H., Bircher J., Weiber M., Scholl E., Preisig R., r974. Bile formation in the intact pig. Am. J. Vet. Res., 35, 1203-1 208.

ForSGREn E., I930. 24 Stunden Variationen der Gallensekretion. Skand. Arch. Physiol., 59, 21 7-225.

Grenier J., Delage J., Ig6o. Section expérimentale des canaux pancréatiques chez le Chien. Ses répercussions sur le pancréas, les voies biliaires et le parenchyme hépatique. $C$. $R$. Soc. Biol., 154, I6I4-I6I6. 
Hopton D. S., 1973. The influence of the vagus nerves on the biliary system. Brit. J. Surg., 60, 2 I $6-2 \mathrm{I} 8$.

JOSEPHSON B., I94I. The circulation of the bile acids in connection with their production, conjugation and excretion. Physiol. Rev., 21, 463-486.

KOSter H., Shapiro A., IERner H., i936. On the rate of secretion of bile. Amer. J. Physiol., $115,23-26$.

LAPLACE J. P., 1972. Circulation sanguine croisée chronique chez le Porc. Technique chirurgicale, entretien, validité. J. Physiol., Paris, 64, 165-172.

IAPI ACE J. P., I976a. L'excrétion biliaire chez le Porc. I) Electromyographie des voies biliaires extra hépatiques. Rec. Med. Vet., 152, 33-43.

LAPLACE J. P., I976b. L'excrétion biliaire chez le Porc. 2) Electromyographie et dynamique de l'excrétion de bile. Rec. Med. Vet., 152, 40I-4II.

LAPLACE J. P., Tomassone, R., I97o. Évacuation gastro-duodénale chez le Porc. Fistulation chronique par voie thoracique extra pleurale. Recherche d'une technique d'analyse mathématique de l'évacuation. Ann. Zootech., 19, 303-332.

Schmid' C. R., BeAzel. J. M., Berman A. C., Ivy A. C., Atrinson A. J., I939. Studies on the secretion of bile. Amer. J. Physiol., 126, I20-I35.

Shriner W., r967. Studies on the common duct. Pressures and mechanisms. Amer. J. Gastroent., 48, $198-203$.

VOGEL G., GRotT W., KüRTEN M., x966. Die Bedeutung des Angebots von Galle füir die aüssere Sekretion des Pankreas. Pflügers Arch. Ges. Physiol., 288, ro3-108.

WyatT A. P., BELZER F. O., DUNPHX J. E., I967. Malfunction without obstruction of the common bile duct. Am. J. Surg., 113, 592-598. 